\title{
Potential protective effects of breast milk and amniotic fluid against novel coronavirus SARS-CoV-2.
}

\author{
April Rees ${ }^{1}$, Steve Turner ${ }^{2}$, and Catherine Thornton ${ }^{1}$ \\ ${ }^{1}$ Swansea University \\ ${ }^{2}$ University of Aberdeen
}

September 24, 2021

Potential protective effects of breast milk and amniotic fluid against novel coronavirus SARSCoV-2.

Authors: April Rees ${ }^{1}$, Stephen Turner ${ }^{2}$, Catherine Thornton ${ }^{1 *}$

${ }^{1}$ Institute of Life Science, Swansea University Medical School, Swansea, Wales, UK, SA2 8PP

${ }^{2}$ University of Aberdeen, The Institute of Applied Health Sciences, Aberdeen, Scotland, UK AB24 3FX

Disclosure: The authors report no conflict of interest.

Funding: This work was supported by the EPSRC Impact Acceleration Account at Swansea University and the Welsh Government Sêr Cymru III Tackling COVID-19 initiative.

${ }^{*}$ Corresponding author:

Professor Cathy Thornton

ILS1, Swansea University Medical School

Singleton Campus

Swansea University

Swansea, Wales, UK

SA2 8PP

Telephone: 01792602122

Email: c.a.thornton@swansea.ac.uk

Keywords: Breast milk, amniotic fluid, SARS-CoV-2, neonate

Word count: 769

To the Editor,

Objective

Infection of pregnant women during previous coronavirus mediated pandemics such as Severe Acute Respiratory Syndrome (SARS) and Middle East Respiratory Syndrome (MERS), were associated with high rates of fetal and maternal demise. However, the characteristics of COVID-19 in pregnant and non-pregnant women are very similar and while severe COVID-19 in pregnancy brings increased risk of preterm birth and intensive care admission ${ }^{1}$ most pregnant women, be they symptomatic or asymptomatic for SARS-CoV-2 infection, 
do not experience severe complications in pregnancy ${ }^{2}$. Similarly, cases of SARS-CoV-2 vertical transmission are rare and there is low risk of serious disease for the neonate ${ }^{3}$. Understanding the mechanisms of resilience against severe COVID-19 in pregnant women and the newborn are critical to ensure ongoing vigilance in care and to provide insight into disease pathogenesis and therapeutic opportunities.

Angiotensin-converting enzyme 2 (ACE2), CD26, CD147 and neuropilin-1 (NRP-1) are some of the key molecules identified as contributing to the entry of coronaviruses such as SARS-CoV-2 into human cells ${ }^{4}$. There is immense interest in these and viral entry facilitating proteases such as transmembrane serine protease-2 (TMPRSS2) as therapeutic targets for limiting infection. Soluble forms of viral entry receptors have been postulated to act as viral traps for SARS-CoV-2 by preventing interaction of the virus with membrane-bound forms ${ }^{5}$. We predict that elevation of these at the maternal-fetal interface contributes to the lack of vertical transmission and limits severity of disease from SARS-CoV-2 infection in the fetus and neonate.

Hypothesis : Soluble (s) ACE2, sCD26, sCD147 and sNRP-1 are elevated at the maternal-fetal interface.

\section{Study Design}

Samples of amniotic fluid (AF) collected at $>37$ weeks of gestation (North of Scotland Research Ethics Committee: 06/S0801/77) and breast milk (BM) collected at $2(2 \mathrm{~W})$ and 6 weeks $(6 \mathrm{~W})$ postpartum (Wales Research Ethics Committee: 2004/024) archived prior to the COVID-19 pandemic were available. All samples were rendered cell free by centrifugation and breast milk defatted prior to arhciving. ELISAs (DuoSet, R\&D Systems) were performed to determine the concentration of sACE2 (catalogue number: DY933), sCD26 (DY1180), sCD147 (DY972) and sNRP-1 (DY3870) in AF and 2W and 6W BM (Figure 1A). Statistics were performed using a one-way ANOVA; a p value of $<0.05$ was deemed significant. Immunoblotting analysis (ACE2 (15983), CD26 (67138), Cell Signalling Technologies; CD147 (AF972), Bio-Techne; NRP-1 (ab81321), Abcam) was used to confirm findings from the ELISAs and reveal different isoforms (Figure 1B).

\section{Results}

All samples were rich in the molecules of interest; BM had significantly higher sACE2 and sNRP-1 but lower sCD26 than AF. Different isoforms of sACE2, sCD26 and sCD147 existed in AF compared to BM and might provide information about the origins of soluble forms of these mediators ${ }^{6}$ and dictate their biological function ${ }^{7}$. For example, the shorter isoform of ACE2 lacks SARS-CoV-2 spike high-affinity binding sites ${ }^{7}$ so AF sACE2 might be less efficient in acting as a viral decoy. The lower molecular weight (MW) form of CD26 is typically associated with its membrane-bound form (which can be shed) compared to the higher MW of the naturally occurring soluble form ${ }^{6}$ suggesting that BM sCD26 originates by shedding from mammary epithelial cells whereas AF contain the naturally occurring soluble form. As these two isoforms have shared and distinct properties there are likely different functional effects of sCD26 in AF and BM that might include the ability to bind SARS-CoV-2. CD147 evidently has various degrees of glycosylation, with BM exhibiting numerous isoforms in comparison to the one isoform observed in AF. The level of glycosylation is correlated to CD147 function, with low glycosylation (LG) forms being unable to self-aggregate and stimulate matrix metalloproteinase (MMP) induction which the high glycosylation (HG) forms are capable ${ }^{8}$. SARS-CoV-2 severity has been associated with an increase in MMP activity ${ }^{9,10}$. The presence of the LG forms in the BM and AF suggests a tighter regulation of MMP activity, potentially preventing severe disease.

\section{Conclusion}

These results suggest that both $\mathrm{AF}$ and $\mathrm{BM}$ are rich in soluble forms of at least some of the molecules that regulate SARS-CoV-2 host cell entry and could help protect the fetus and neonate from infection by acting as decoy receptors and contributing to innate immune protection in the gastrointestinal and, in the case of AF, respiratory tracts (Figure 1C). Such a mechanism might also be relevant for other viral infections. Future work should focus on evaluating if $\mathrm{AF}$ and $\mathrm{BM}$ inhibit viral entry into host cells and comparing the relative efficacy of different isoforms. This could reveal a novel protective mechanism for the baby against SARS$\mathrm{CoV}-2$ infection and/or severe disease and provide further evidence for a protective role for breastfeeding. 
April Rees ${ }^{1}$, Stephen Turner ${ }^{2}$, Catherine Thornton ${ }^{1}$

${ }^{1}$ Institute of Life Science, Swansea University Medical School, Swansea, Wales, UK, SA2 8PP

${ }^{2}$ University of Aberdeen, The Institute of Applied Health Sciences, s, Aberdeen, Scotland, UK AB24 3FX References

1. Martinez-Perez O, Prats Rodriguez P, Muner Hernandez M, et al.The association between SARS-CoV2 infection and preterm delivery: a prospective study with a multivariable analysis. BMC Pregnancy and Childbirth 2021; 21 : 273.

2. Vousden N, Bunch K, Morris E, et al. The incidence, characteristics and outcomes of pregnant women hospitalized with symptomatic and asymptomatic SARS-CoV-2 infection in the UK from March to September 2020: A national cohort study using the UK Obstetric Surveillance System (UKOSS). PLOS ONE 2021; 16 : e0251123.

3. Gale C, Quigley MA, Placzek A, et al. Characteristics and outcomes of neonatal SARS-CoV-2 infection in the UK: a prospective national cohort study using active surveillance. The Lancet Child $\&$ Adolescent Health 2021; 5 : 113-121.

4. Radzikowska U, Ding M, Tan G, et al. Distribution of ACE2, CD147, CD26, and other SARS-CoV-2 associated molecules in tissues and immune cells in health and in asthma, COPD, obesity, hypertension, and COVID-19 risk factors. Allergy 2020; 75 : 2829-2845.

5. Monteil V, Kwon H, Prado P, et al. Inhibition of SARS-CoV-2 Infections in Engineered Human Tissues Using Clinical-Grade Soluble Human ACE2. Cell 2020; 181 : 905-913.e907.

6. Duke-Cohan JS, Morimoto C, Rocker JA, Schlossman SF. A Novel Form of Dipeptidylpeptidase IV Found in Human Serum: Isolation, Characterization, and Comparison with T Lymphocyte Membrane Dipeptidylpeptidase IV (CD26). Journal of Biological Chemistry1995; 270 : 14107-14114.

7. Blume C, Jackson CL, Spalluto CM, et al. A novel ACE2 isoform is expressed in human respiratory epithelia and is upregulated in response to interferons and RNA respiratory virus infection.Nature Genetics 2021; 53 : 205-214.

8. Tang W, Chang SB, Hemler ME. Links between CD147 function, glycosylation, and caveolin-1. Molecular biology of the cell2004; $15: 4043-4050$.

9. Solun B, Shoenfeld Y. Inhibition of metalloproteinases in therapy for severe lung injury due to COVID-19. Medicine in Drug Discovery2020; 7 : 100052.

10. Ueland T, Holter JC, Holten AR, et al. Distinct and early increase in circulating MMP-9 in COVID-19 patients with respiratory failure. The Journal of infection 2020; 81 : e41-e43.

Figure Legend 
A
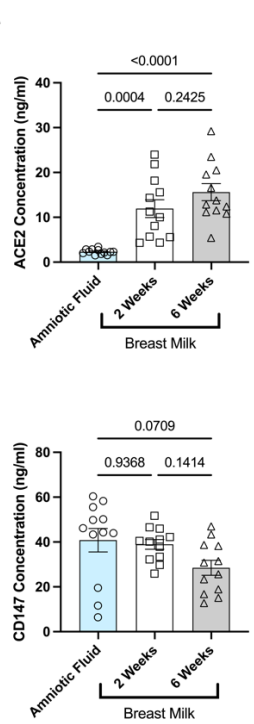
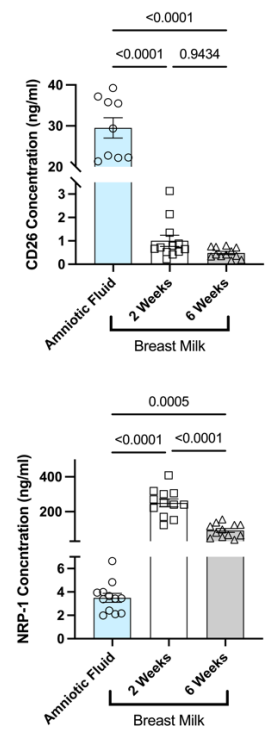

B

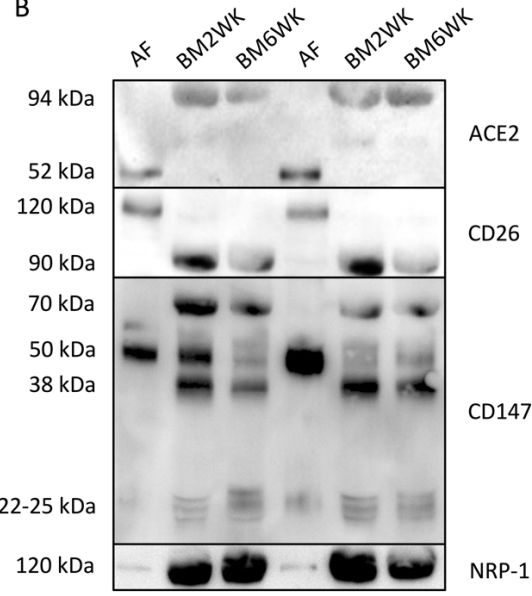

C

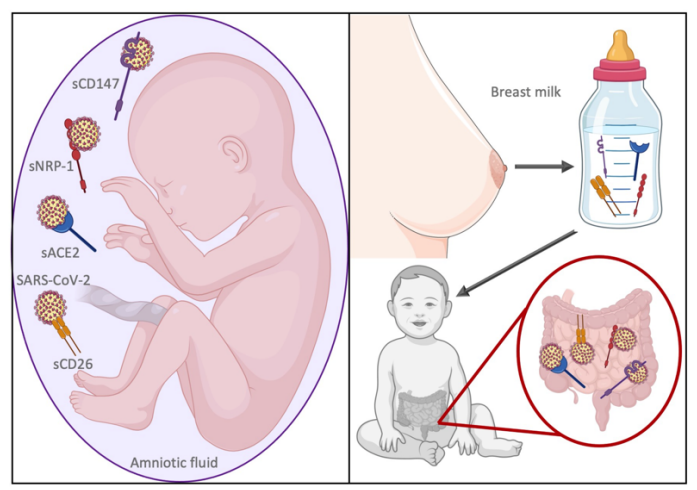

Figure 1: Presence of soluble SARS-CoV-2 host cell entry associated molecules in amniotic fluid (AF) and breast milk (BM) at 2- and 6-weeks postpartum. (A) Soluble molecules (ACE2, CD26, CD147 and NRP-1) were measured in AF and BM using ELISAs. (B) The presence of these soluble molecules was confirmed with Western Blotting and isoforms were identified. (C) The presence of these soluble molecules in AF and BM which the baby ingests and in the case of amniotic fluid also aspirates may confer resistance to SARS-CoV-2 infection and/or severe COVID-19. (The images in (C) were created with BioRender). 